\title{
Erratum to: Linking Corporate Policy and Supervisory Support with Environmental Citizenship Behaviors: The Role of Employee Environmental Beliefs and Commitment
}

\author{
Nicolas Raineri ${ }^{1} \cdot$ Pascal Paillé $^{1}$
}

Published online: 20 April 2016

(C) Springer Science+Business Media Dordrecht 2016

\section{Erratum to: J Bus Ethics DOI 10.1007/s10551-015-2548-x}

\section{Presentation of the research model}

The results are correct, but there is a mistake in the article that reduces the understanding of the research model and of the results.

In the following sentences from the article, the word "person" should be replaced by the word "group", which modifies the interpretation of the research model and of the results:

Page 2: Incorrect: The model involved hypotheses at both the within-person and between-person levels.

Correct: The model involved hypotheses at both the within-group and between-group levels.

Figure 1 on page 3: Incorrect: Between-person level“" and "Within-person level.

Correct: Between-group level" and "Within-group level
Page 11: Incorrect: The research model involved hypotheses at both the within-person and between-person levels, and was tested in two steps. First, within-person Hypotheses 1-4 were tested using the full data sample... Second, between-person Hypotheses 5a and 5b were analyzed using the multi-sample invariance technique.

Correct: The research model involved hypotheses at both the within-group and between-group levels, and was tested in two steps. First, within-group Hypotheses 1-4 were tested using the full data sample... Second, betweengroup Hypotheses $5 \mathrm{a}$ and $5 \mathrm{~b}$ were analyzed using the multi-sample invariance technique.

Pages 11 and 13: Under the Title "Hypothesis Testing" (p.11), the following subtitles are also incorrect

Incorrect: Within-Person Level (p. 11), and BetweenPerson Level (p. 13).

Correct: Within-Group Level (p. 11), and BetweenGroup Level (p. 13).
Nicolas Raineri

raineri.n@gmx.com

Pascal Paillé

pascal.paille@fsa.ulaval.ca

1 Department of Management, Faculty of Business Administration, Laval University, Pavillon Palasis-Prince, Local 1638, 2325, rue de la Terrasse, Quebec, QC G1V 0A6, Canada 